\title{
Introduction to the Minitrack on Frontiers in AI and Software Engineering (FAISE'18)
}

\author{
Rick Kazman \\ University of Hawaii \\ kazman@hawaii.edu
}

\author{
Tim Menzies \\ Computer Science, NC State \\ tim@,menzies.us
}

\begin{abstract}
Welcome to FAISE'18, the first HICCS minitrak on Frontiers in AI and Software Engineering. This minitrack welcomes papers not only on the application of AI techniques to SE problems but also the application of SE techniques to AI problems
\end{abstract}

\section{Introduction}

The motivation for the meeting of FAISE'18 is the following question:

"What is role of AI in the future of SE?"

In the last decade AI has gone from a minor side technology to one of the dominate drivers in technological innovation on the planet. If we look ahead 5,10,20 years, will there even be programmers? Or will software engineering become (say) dropping a specification into some use AIdriven cloud machine that returns for our inspection and selection, hundreds of variants of potential solutions?

So it is time to ask, in this age of Google AI, and text miners that can read our texts better than us, and program synthesis agents that can code better than us, and data miners that can look at more information that we can:

- What is the future role of software engineers?

- What parts of SE should we willingly and quickly discard and what parts should be always keep and preserved into the future?

Note that FAISE'18 is NOT a venue requiring rigorous and tedious proof and/or evaluation of the submitted ideas (since, certainly, there are enough of those). Instead, FAISE'18 is a venue to explore "over the horizon" research ideas. Here, we brainstorm; speculate; review past results at an ultra-high-level; while at the same time try to imagine the future and our role in creating it.

\section{Our Papers}

In response to the challenge of our Carol V. Alexandru and Harald C. Gall write in Of Cyborg Developers and Big Brother Programming AI that many day-to-day activities in software engineering (such as bug triaging, reverting regressions, or even implementing code for properly scoped problems) could possibly be automated, but we lack the necessary monitoring tools to capture all relevant information. Hence, these explore ways to capture that required background knowledge.

FAISE' 18 is hardly the first venue to explore the interaction of AI and SE. In Performance Characterization of State-Of-The-Art Deep Learning Workloads on an IBM "Minsky" Platform, Mauricio Guignard et al. discuss how to lever's IBM AI tools to address important SE tasks.

In other work, Muhammad Karim et al. look into how to combine large scale human and artificial intelligence. In Learn or Earn? - Intelligent Task Recommendation for Competitive Crowdsourced Software Development, they discuss the role of crowdsourcing to support intelligent decision support in SE.

Finally, in our most speculative work, Nicolas Guelfi challenges us to Please ... . draw me a Software Engineer. In this over-the-horizon vision of the future, Guelfi imagines replacing "software engineers" with "senseware" engineers that build physical space and time augmentations that adapt to human direct and indirect needs. 